\section{Linking tissues to disease}

A recent analysis in Nature Genetics combines genome-wide association study summary statistics with gene expression data to identify the contribution of different cell types and tissues to disease.

In a paper published in Nature Genetics, Finucane et al. combine genome-wide association study (GWAS) summary statistics with gene expression data to identify disease-relevant cell types and tissues.

Using five gene expression data sets from humans and mice across multiple tissues, the team set out to investigate whether a specific tissue was enriched for a given trait. Linear regression of genome-wide associations of genetic variants with disease phenotypes enabled the group to evaluate the contributions of specific tissues and cell types to trait heritability and disease in a technique they call linkage disequilibrium score regression applied to specifically expressed genes (LDSC-SEG).

In total, the researchers investigated 48 diseases and traits. For 34 of them, at least 1 tissue showed significant contribution to the trait. This approach helped to confirm known biological associations; for example, psychiatric traits were associated with brain-specific gene expression.

The use of multiple tissues also enabled the team to look at contributions from different sources to disease. The analysis of immunerelated traits and gene expression showed enrichment for variants associated with bipolar disorder and schizophrenia, alongside immunological diseases, but not other psychiatric diseases.

The group also investigated the association of genetic variants with chromatin data from the Roadmap Epigenomics and ENCODE
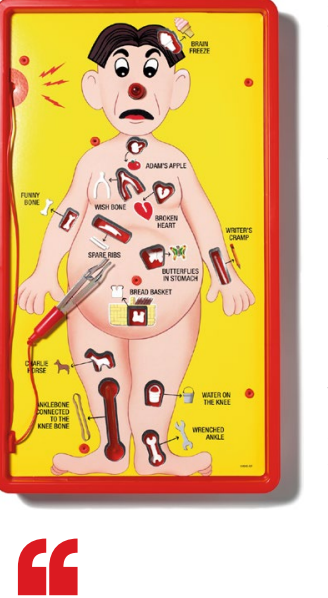

analysing

polygenic

signals in

multiple tissues

... was able

to identify the

contribution of

many tissues

and cell types

to a disease

5

projects to determine the role that genome architecture may have in disease. The analysis of chromatin data included some cell types that were absent from the gene expression data. In one case this enabled the detection of an enrichment for neurological tissues in a migraine data set including both migraine with and without aura; this enrichment was absent when looking only at migraine without aura, suggesting that different tissues may be relevant for different migraine subtypes.

By analysing polygenic signals in multiple tissues, the group was able to identify the contribution of many tissues and cell types to a disease. While these associations will be difficult to evaluate in vitro and in vivo, as the authors caution, this study helps to lay the groundwork for future investigations into the mechanisms of disease pathology.

Ross Cloney, Senior Editor, Nature Communications

ORIGINAL ARTICLE Finucane, H. K. et al. Heritability enrichment of specifically expressed genes identifies disease-relevant tissues and cell types. Nat. Genet. 50, 621-629 (2018)

\title{
Taking aim at transcriptional regulator targets
}

A paper in Science reports a strategy that combines rapid chemical-genetic perturbation with measurements of immediate gene expression changes to identify targets of transcriptional regulators.

Classic functional genomics assays, whereby changes in gene expression are monitored after a factor of interest is disrupted, cannot distinguish between direct and indirect effects. Muhar et al. now present a strategy that combines rapid chemical-genetic perturbation with measurements of immediate gene expression changes to preclude indirect effects.

The authors set out to identify targets of transcriptional regulators such as bromodomain-containing protein 4 (BRD4), a member of the bromodomain and extra-terminal motif (BET) protein family. BRD4 is reversibly bound by BET inhibitors (BETi), which prevents its interaction with acetylated histones and leads to repression of its target genes.
BRD4 expression was perturbed in $\mathrm{K} 562$ cancer cells using the auxin-inducible degron (AID) system, whereby the degradation of AID-tagged target proteins can be rapidly induced. The authors then applied an optimized version of thiol (SH)-linked alkylation for the metabolic sequencing of RNA (SLAM-seq), a previously established genome-wide method for measuring nascent mRNAs. They observed overall transcriptional downregulation and accumulation of RNA polymerase II (Pol II) at transcription start sites, confirming that BRD4 exerts transcriptional control by releasing paused Pol II.

The effects of high-dose BETi treatment mirrored those observed after BRD4 degradation, but applying lower therapeutically active BETi doses revealed a selective transcriptional response that affected only a small set of hypersensitive genes. Applying their strategy to the hypersensitive target MYC, the team introduced an

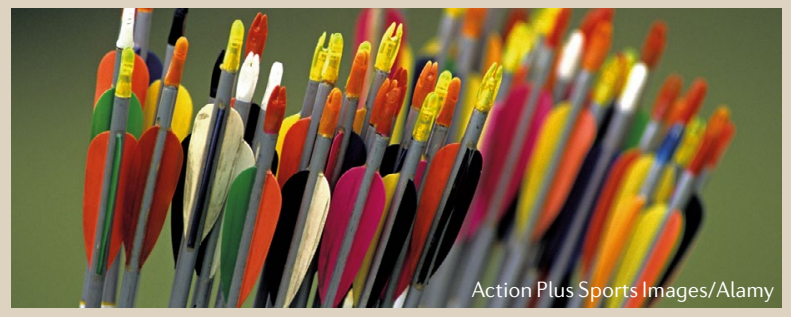

AID-tag into the endogenous MYC locus of two independent cancer cell lines and used SLAM-seq to quantify

a strategy that combines rapid chemical-genetic perturbation with

measurements of immediate gene expression changes

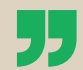
changes in mRNA output following MYC degradation. In contrast to BRD4, the transcriptional responses observed after MYC degradation in both cell lines were highly specific rather than global, suggesting that MYC activates the transcription of specific target genes rather than functioning as a general transcriptional activator or repressor.

Taken together, mapping direct transcriptional responses to the degradation of AID-tagged proteins provides a scalable method to explore the gene regulatory landscape.

Linda Koch

ORIGINAL ARTICLE Muhar, M. et al. SLAM-seq defines direct gene-regulatory functions of the BRD4-MYC axis. Science https://doi.org/10.1126/ science.aao2793 (2018) 